\title{
Why are clinical costs so high?
}

Trial requirements and inefficiencies are driving up the costs of clinical development.

\section{Simon Frantz}

The figure of $\$ 802$ million needs no introduction. Some may question the value of this figure for the average cost of drug development from the Tufts Center for the Study of Drug Development. But one theme emerges when the total cost is broken down into its component parts: the cost of clinical development has leapfrogged preclinical costs to become the most expensive aspect of drug development.

According to the latest study published by Joseph DiMasi et al., the average clinical cost of approved compounds has more than quadrupled since their last study of drug development costs in 1991 (DiMasi, J. A., Hansen, R. W. \& Grabowski, H. G. J. Health Econ. 22, 151-185 (2003)). The average clinical cost, inclusive of drug failure and time costs, for 68 randomly sampled therapeutic compounds that entered clinical trials between 1983-1994 was US \$467 million (year 2000 \$), compared with US \$104 million for compounds that entered clinical trials between 1970-1982. During this time, preclinical costs only rose from US \$214 million to US $\$ 335$ million (see figure).

"People don't seem surprised by the rise in clinical costs," says Joseph DiMasi, Director of Economic Analysis at the Tufts Center for the Study of Drug Development.

DiMasi says there could be many reasons. "More treatments were being developed for chronic and degenerative diseases or conditions associated with them, which are

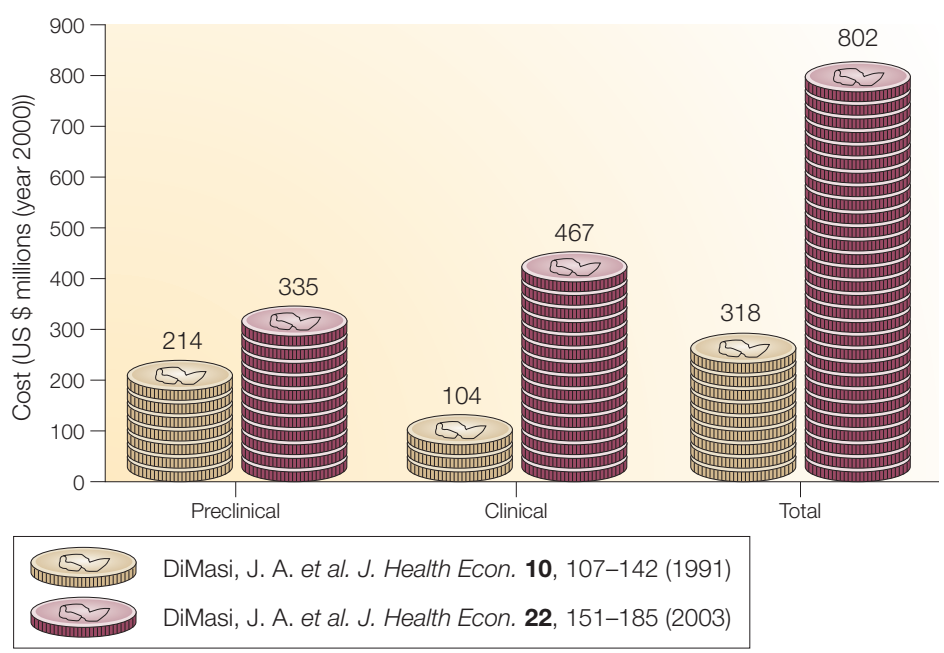

The average preclinical, clinical and total costs, inclusive of drug failure and time costs, of approved compounds.

more costly to test because they require more patient care, longer patient-monitoring periods, and more and larger trial sizes," he says. And greater numbers of drugs coming through the pipeline means more resources are needed for patient recruitment.

Also, for some conditions, such as cardiovascular disease, more drugs on the market means that drugs in development need more compelling evidence to affect clinical practice. So, increasingly, trials comparing treatments versus competitors rather than placebo are carried out, which are costlier and usually need to be more highly powered to show a significant effect.

In addition, the need for new drugs, in particular 'me-too' drugs, to make their mark in a crowded market often means that they are tested in larger, lower-risk populations. And harder clinical end points, such as death or myocardial infarction, rather than the softer end points used in earlier trials, such as lowering blood pressure, are increasingly required.

Figures from CenterWatch, a Boston-based company that collects clinical trial information, show that the number of active clinical studies worldwide increased from 3,275 in 1994 to 3,900 in 2002. The median evaluable patients per New Drug Application has increased from 3,233 in 1985-1988 to 5,621 in 1998-2001.

Clinical phase lengths have fluctuated between 1980-2001, briefly peaking in 1994-1995 (Reichert, J. M. Nature Rev. Drug Discov. 2, 695-702 (2003)). Overall, they have not escalated in a dramatic fashion, although some therapeutic areas, such as antineoplastics, saw large increases in 
- mean clinical phase times from around 90 to 150 months in the early 1990s.

The magnitude of the increase in trial size numbers does not intuitively imply a quadrupling of costs. But there are also hidden costs, says Kenneth Getz, CEO of CenterWatch. For example, the proportion of clinical studies that are delayed by more than a month has risen from $60 \%$ in 1997 to $72 \%$ in 2003 . As well as adding to the clinical costs, figures from CenterWatch show that every day of prescription drug sales lost due to delays called 'opportunity costs' - takes an average toll of US \$1.3 million, with losses for blockbuster drugs reaching as much as US $\$ 4-5$ million a day.

A principal reason for delays seems to be patient recruitment. "Half a billion dollars are spent by companies on patient recruitment," says Getz. And 38\% of 405 trial sites surveyed by a US Investigative Site Survey in 2003 strongly agreed that slow patient recruitment delayed clinical development. Sites also highlighted contract and budget negotiations, protocol amendments, legal review processes, and institutional review board reviews and approvals as reasons for delays.

\section{Pinning down the reasons for the increase in clinical costs seems to be extremely tricky}

Clinical development also incurs other costs. Generally the greatest cost is for enrolling subjects. But hiring personnel from drug companies, drug-production costs, payments to investigators, ethics committees and regulatory authorities, and equipment purchases for laboratories and centres all add to the overall financial burden.

A taskforce called the Pharmaceutical Industry Competitiveness Task Force (PICTF), which was set up by the United Kingdom government to examine the state of the UK pharmaceutical industry, found that one of the most important areas for improvement is in approving and overseeing clinical trials. "These are not headline-grabbing issues, but they are all very important," says Richard Ley, at the Association of the British Pharmaceutical Industry.

Pinning down the reasons for the increase in clinical costs seems to be extremely tricky, which in part results from the scope and complexity of today's clinical trials. A scan of pharmaceuticalrelated conferences shows a multitude of meetings devoted to improving the efficiency of preclinical technologies, but a relative paucity of those devoted to the analysis of clinical costs. Given that clinical trials are now the most expensive area of drug development, shouldn't getting to the root of the problem be a top priority? 